\title{
Acoustic streaming enhanced mass transfer at a wall
}

\author{
Nouhayla El Ghani ${ }^{a}$, Sophie Miralles ${ }^{\mathrm{a}, *}$, Valéry Botton ${ }^{\mathrm{a}}$, Daniel Henry ${ }^{\mathrm{a}}$, Hamda Ben \\ Hadid $^{\mathrm{a}}$, Benoît Ter-Ovanessian ${ }^{\mathrm{b}}$, Sabrina Marcelin ${ }^{\mathrm{b}}$ \\ ${ }^{a}$ Laboratoire de Mécanique des Fluides et d'Acoustique, CNRS/Université de Lyon, Ecole Centrale de \\ Lyon/Université Lyon 1/INSA Lyon, ECL, 36 Avenue Guy de Collongue, 69134 Ecully Cedex, France \\ ${ }^{b}$ Université de Lyon, INSA Lyon, UMR CNRS 5510, Laboratoire MATEIS, F-69621 Villeurbanne \\ Cedex, France
}

\begin{abstract}
The influence of an impinging acoustic streaming jet on wall mass transfer is studied both experimentally and numerically. The idea is to show that acoustically-driven jets generated by ultrasounds can be used to enhance transfer phenomena at a distance, by creating localized friction zones. An experimental setup has been developed consisting in a cavity containing an electrolytic solution of $\left[\mathrm{Fe}(\mathrm{CN})_{6}\right]^{4-} /\left[\mathrm{Fe}(\mathrm{CN})_{6}\right]^{3-}$. A jet forced by an ultrasound beam impinges on the upper wall instrumented with electrodes, at which the mass transfer influenced by the streaming is measured by electrochemical technics. Numerical simulations of the flow and mass transfer in the same configuration are also performed. A significant enhancement of the mass transfer at the electrodes (represented by the Sherwood number $S h$ ) with the injected acoustic power (quantified by the acoustic Grashof number $G r_{\mathrm{ac}}$ ) is observed. An order of magnitude of the expected Sherwood number and friction coefficient is proposed on the basis of the Leveque law and momentum budget considerations. Scaling laws involving both experimental and numerical mass transfer at the electrodes $(S h)$, numerical wall shear stress and injected power $\left(G r_{\text {ac }}\right)$ are finally derived.
\end{abstract}

Keywords: Mass transfer, Acoustic streaming, Impinging jets, Polarometry, Chronoamperometry

\section{Introduction}

We report an experimental work on the enhancement of local mass transfer at a wall using an ultrasound beam. Numerous industrial processes imply heat and mass transfer at a wall at different scales and could be concerned by this investigation. Let

5 us already mention the known influence of ultrasounds on electrochemical processes such as electrodeposition [1] and on crystal growth from a melt and solidification of metallic alloys [2, 3, 4, 5, 6, 7, 8], but also in vivo mass transfer enhancement in living [9] and human beings (improving the efficiency of an active substance with a local target within

\footnotetext{
* Corresponding author

Email address: sophie.miralles@insa-lyon.fr (Sophie Miralles )
} 
the body) and miscellaneous medical applications [10, 11, 12]. Of course, wall heat transfers can also be enhanced using ultrasounds [13].

The mentioned mechanism for mass transfer enhancement is usually acoustic streaming: progressive waves in the acoustic beam drive a quasi-steady flow in the direction of wave propagation; this flow consequently yields some convection which enhances mass transfer. This streaming is due to non-linear effects in the momentum equations for a 15 compressible fluid; a model can be derived by averaging these equations over the acoustic period [14, 15, 16] to account for the steady influence of the rapidly oscillating flow associated to the acoustic wave. The equations thus obtained are simply the Navier-Stokes equations for an incompressible fluid, in which a space dependent acoustic force term $f_{\mathrm{ac}}=\frac{2 \alpha I_{\mathrm{ac}}}{c}$ is introduced $\left(\alpha\left(\mathrm{m}^{-1}\right)\right.$ is the acoustic attenuation depending on the fluid

2o and the acoustic frequency, $I_{\mathrm{ac}}\left(\mathrm{W} \cdot \mathrm{m}^{-2}\right)$ is the space dependent acoustic intensity, and $c$ (m.s $\left.{ }^{-1}\right)$ is the sound speed in the working fluid). $I_{\mathrm{ac}}$, and then the force $f_{\mathrm{ac}}$, are obtained as the solution of the acoustic propagation problem. Note, however, that, when considering heat and mass transfer, it has been formerly observed that a rapidly oscillating periodic flow can also drive additional time-averaged transfers [17, 18, 19] often considered as additional diffusion. Such additional transfers are often considered as negligible without any justification in numerical studies $[20,21,22,23$.

Electrochemical techniques have already been used to study the influence of ultrasound on wall mass transfer [24, 25]. These former experimental investigations pointed out the importance of both the hydrodynamical and cavitation effects and showed that

so the use of an acoustic source of a few $\mathrm{kHz}$ operating at high power (up to $600 \mathrm{~W}$ ) can significantly impact the mass transfer. However, no information is given on the coherence of the ultrasound acoustic beam used and on the characteristics of the flow thus generated. Several effects can be expected when using relatively high power ultrasounds, in particular thermal effects [20, 21] and cavitation effects [26] when using High Intensity

35 Focused Ultrasounds (HIFU), or even atomization when considering a free surface. In the present case featuring a plane (unfocused) transducer, thermal and cavitation effects are expected to be negligible. This is all the more true as the frequency is chosen too high for cavitation to occur, but not high enough for thermal effects due to attenuation in the medium to be significant [16]. In the present work, we want to use ultrasound at

4o higher frequencies, while avoiding cavitation. The objective is that the acoustic beam remains coherent over sizeable lengths (decimeters to meters), in order to be able to act at great distances. Using higher frequencies makes it also possible to drive significant flows with very low powers, due to the increase of the attenuation coefficient $\alpha$ as the square of the frequency.

45 Our main concern is then to understand how mass transfer is enhanced by an acoustically-driven flow impinging on a wall. To address this question, we hereunder present our measurements of mass flux with electrochemical techniques under the influence of a tilted (non normal to the wall) ultrasonic beam. We derive an observational link between the mass flux at the wall and the acoustic power in the acoustic beam. In addition, we present

so numerical simulations of the flow and mass transfer in the same configuration, allowing to show the correlation between mass flux and local shear stress, following Leveque formula.

Details on the adaptations of the experimental setup are presented in section 2 . Then we focus on the calibration technique using the short time response of the electrochemical cell in section 3 In section 4, we describe the wall mass transfer measurements, enhanced 
55 by the action of the acoustic streaming. The numerical simulations allowing to calculate the wall shear stress and the convecto-diffusive mass transfer are presented in section 5. The mass transfer results obtained both experimentally and numerically are analyzed in section 6 and scaling laws of the mass transfer dimensionless parameter (the Sherwood number) are finally derived.

\section{Experimental setup developed for electrochemical measurements}

The experimental setup (Fig. 1] is very similar to that in [27] designed to investigate the water flow driven by an ultrasonic beam reflecting on a wall. It has been modified in order to conduct electrochemical measurements to estimate the mass flux at this wall. Here, the $2 \mathrm{MHz}$ acoustic beam, generated by a plane $d_{s}=29 \mathrm{~mm}$ diameter transducer

65 (Imasonic $^{\mathrm{TM}}$ ), is oriented with an angle of $\theta=27.5^{\circ}$ with the horizontal to impinge on the top glass-wall, which will be referred to as the instrumented wall. This is shown in Fig. 1 where the acoustic beam axis (called acoustic axis in the following) is plotted as a red line. The glass tank $(155 \mathrm{~mm} \times 260 \mathrm{~mm} \times 182 \mathrm{~mm}$ corresponding to height $\times$ length $\times$ width $)$ is filled with an aqueous solution of $\left[\mathrm{Fe}(\mathrm{CN})_{6}\right]^{4-} /\left[\mathrm{Fe}(\mathrm{CN})_{6}\right]^{3-}$ at a nominal

7o concentration $C^{0}=0.01 \mathrm{~mol} . \mathrm{L}^{-1}$, with $\mathrm{KCl}$ as a support electrolyte. The bottom side of the tank is a large counter electrode made of carbon graphite, covering more than $60 \%$ of the bottom surface, maintained with a PVC frame. A saturated calomel reference electrode $\left(\mathrm{E}_{\mathrm{ref}}\right)$ is also introduced in the solution. Eight platinum electrodes, numbered from 0 to 7 ( $E_{0}$ to $\left.E_{7}\right)$, are mounted on the top wall flush to its lower surface in order

75 to probe wall mass transfer. They will be referred to as the working electrodes. Their nominal diameter is $3 \mathrm{~mm}$; their locations are chosen to span the wall, including sonicated and non-sonicated regions as can be seen in Fig. 1 b. The isovalues of the normalized acoustic force $F_{\text {ac }} / F_{\text {ac,max }}$ at the wall, computed through a linear acoustic propagation model, are also shown in the same figure. Electrodes $E_{2}$ and $E_{3}$ are considered to stand

so within the core of the beam $\left(F_{\text {ac }} / F_{\text {ac,max }}>0.25\right)$, electrodes $E_{1}, E_{5}$ and $E_{6}$ at the periphery of the beam $\left(0.045<F_{\text {ac }} / F_{\text {ac,max }}<0.25\right)$, and electrodes $E_{0}, E_{4}$ and $E_{7}$ outside the beam $\left(F_{\text {ac }} / F_{\text {ac, } \max }<0.045\right)$.

The working electrode, the counter electrode and the reference electrode are wired to a potentiostat (Gamry Instruments REF 600) measuring the current response of the

85 electrochemical cell under a controlled imposed electric potential. In the case of electrochemical reactions at the surface of a non-reactive electrode (as platinum), a huge overvoltage from the free potential (redox potential of the solution) entails a diffusion limitation of the reaction. Measuring the electric current is then a direct measure of the ions mass flux from the bulk to the wall, through the concentration boundary layer. At

9o steady state, the limit current $I_{\lim }$ and the wall mass flux $\phi$ are linked by the following relation:

$$
I_{\lim }=F \phi,
$$

with $F$ the Faraday constant, and $\phi$ can be expressed as

$$
\phi=\left.A D \frac{\partial C}{\partial z}\right|_{z=h}
$$

where $A$ is the surface area of the working electrode where the reaction occurs, $D$ is the molecular diffusion coefficient [28], and $\left.\frac{\partial C}{\partial z}\right|_{z=h}$ is the normal concentration gradient at the wall for the charged species consumed or generated by the reaction. 


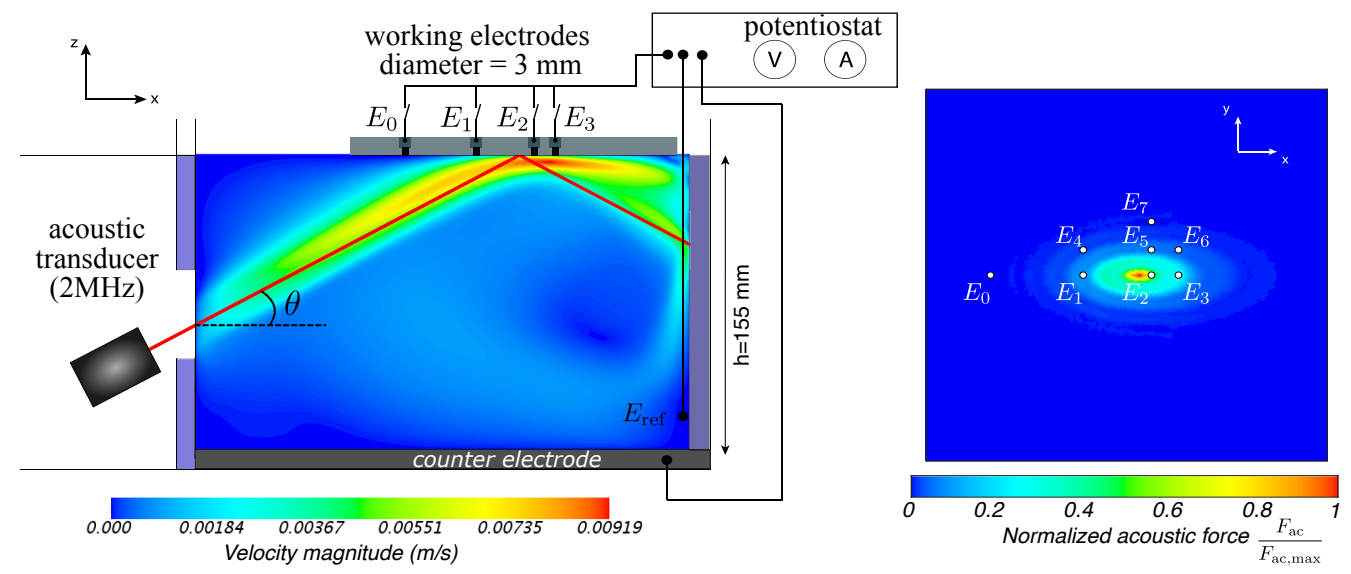

(a)

(b)

Figure 1: (a) 2D schematic side view of the experimental setup with a $2 \mathrm{MHz}$ acoustic source put in water and oriented with an angle of $\theta=27.5^{\circ}$ to shoot on the instrumented plate at the top of the investigated volume. The tank is filled with $\left[\mathrm{Fe}(\mathrm{CN})_{6}\right]^{4-} /\left[\mathrm{Fe}(\mathrm{CN})_{6}\right]^{3-}$ electrolytic solution. The 3 mm diameter platinum working electrodes are connected one at a time to a potentiostat. The counter electrode at the bottom of the cavity and the reference electrode are used to close the circuit and control the electric potential. The solid red line departing from the transducer represents the acoustic beam axis. The super-imposed colormap is the computed velocity field for an acoustic power $P_{\mathrm{ac}}=1.099 \mathrm{~W}$. Details are given in section 5 (b) Geometry of the instrumented top-plate supporting the electrodes. A colormap of the normalized acoustic force $F_{\mathrm{ac}} / F_{\mathrm{ac}, \max }$ has been superimposed on the sketch. The white circles represent the positions of the electrodes.

The principle of the experiment is thus very simple: the incident acoustic beam drives a steady jet impinging on the top wall and flowing then along it by inertia, while the reflected acoustic beam drives a second jet going away from that wall. This so-called yshaped flow, formerly observed in [27, while sweeping the top wall, enhances the supply of reactive species near the working electrodes and consequently improves the reaction rate. Measuring the limit current at each electrode gives us an insight into the dependence of the mass flux with the position in the flow where it is measured and with the applied acoustic power.

We consider that the main difficulties, which are sources of uncertainties, are the mastering of the electrodes surface that effectively reacts with the solution and the precise positioning of the invisible acoustic axis inclined within the cavity, knowing that the distance from the acoustic transducer center to the instrumented wall is large (of about $230 \mathrm{~mm}, 35 \mathrm{~mm}$ before the entrance in the measurement volume and $L_{\mathrm{ac}}=195 \mathrm{~mm}$ in this volume). Moreover, the available space is too small for our hydrophone to measure the position of the acoustic axis directly through acoustic pressure measurements. We thus characterize the acoustic field with the transducer in horizontal position, check that the acoustic axis is well aligned with the $x$ coordinate and finally incline the acoustic source. In that way, we consider that the position of the reflection point, i.e. the intersection of the acoustic axis with the instrumented plane, is known with an accuracy better than 10 mm. More details on the experimental protocol can be found in [29].

In our experiment, the value of the molecular diffusion coefficient $D$ is mostly influenced by the temperature of the solution, which in our case ranged from 25 to $28^{\circ} \mathrm{C}$, but 
without being precisely monitored. According to the literature [28, $D$ is found to vary between $6.6710^{-10}$ and $7.9810^{-10} \mathrm{~m}^{2} \cdot \mathrm{s}^{-1}$. This variation will be taken, within other effects, as a discrepancy in our experimental results. As a consequence, the experimental Schmidt number $S c=\frac{\nu}{D}$, expressing the relative strength of viscous over diffusive effects, spans from 1050 to 1350 . Note that the kinematic viscosity, weakly dependent on the temperature, has been taken as $\nu=8.9110^{-7} \mathrm{~m}^{2} \cdot \mathrm{s}^{-1}$, value at $25.5^{\circ} \mathrm{C}$.

\section{Reference case without acoustic forcing: preliminary calibration using the short time response of the current}

The experimental method used in this electrochemical approach is chronoamperometry. A voltage step is applied to the cell from the open circuit potential/free potential (corresponding to zero electrical current) to $1 \mathrm{~V} / \mathrm{SCE}$ (with respect to the reference electrode $\left.\left(\mathrm{E}_{\text {ref }}\right)\right)$ corresponding to the diffusion plateau to $\left[\mathrm{Fe}(\mathrm{CN})_{6}\right]^{4-} /\left[\mathrm{Fe}(\mathrm{CN})_{6}\right]^{3-}$ redox

130 couple. The current is then monitored during at least $400 \mathrm{~s}$; it has been checked that this time was sufficient to reach a plateau value when the acoustic forcing is applied, but that a longer time is needed in situations without forcing. As the electrochemical reaction takes place, the oxidant $\left[\mathrm{Fe}(\mathrm{CN})_{6}\right]^{4-}$ is quickly consumed with the formation of a concentration boundary layer close to the working electrode. After this transient 135 regime, the reaction is limited by diffusion processes carrying $\left[\mathrm{Fe}(\mathrm{CN})_{6}\right]^{4-}$ ions to the platinum working electrode.

Despite the apparent simplicity of the protocol, special care is required to ensure good measurements. The mounting of the electrodes is especially important, as the current within the cell depends on the area of the working electrode in contact with

140 the electrolytic solution. In our case, the 8 platinum electrodes of the setup have been manually fitted in the glass plate, so that the sealing at the level of the electrodes with varnish might not be perfect, as well as the flushness of each electrode with the glass plate. A second difficulty is that purely diffusive conditions, which should serve as a reference for any investigation of mass transfer enhancement and allow a calibration at

145 each electrode, are never achieved in this large volume of solution due to convection. We overcome these difficulties by considering the transient (short time) response to the applied potential-step. A typical response obtained in our setup is given in Fig. 2. After an initial peak, a clear tendency is observed at intermediate times, typically between $6 \mathrm{~ms}$ and $30 \mathrm{~s}$. This short time response, which occurs before any boundary layer can

150 develop, is well described by the solution of a $1 \mathrm{D}$ transient diffusion problem in a semiinfinite medium with an initial step in concentration at the electrode wall [30. The concentration-step is assumed to correspond to the concentration $C^{0}$ in $\left[\mathrm{Fe}(\mathrm{CN})_{6}\right]^{4-}$ (the reducing component) initially present in the bulk of the solution. The equation giving the instantaneous current density is often referred to as the Cottrell relation and expressed as:

$$
\frac{I(t)}{A}=\frac{F \sqrt{D} C^{0}}{\sqrt{\pi t}} .
$$

Using Eq. (3) with $D=6.6710^{-10} \mathrm{~m}^{2} \cdot \mathrm{s}^{-1}$ and $C^{0}=0.01 \mathrm{~mol} \cdot \mathrm{L}^{-1}$, a least mean squares fit of the transient experimental data gives the value of the effective area $A_{\text {eff }}$ for each electrode. The lower value of fluid temperature has been considered here, given 


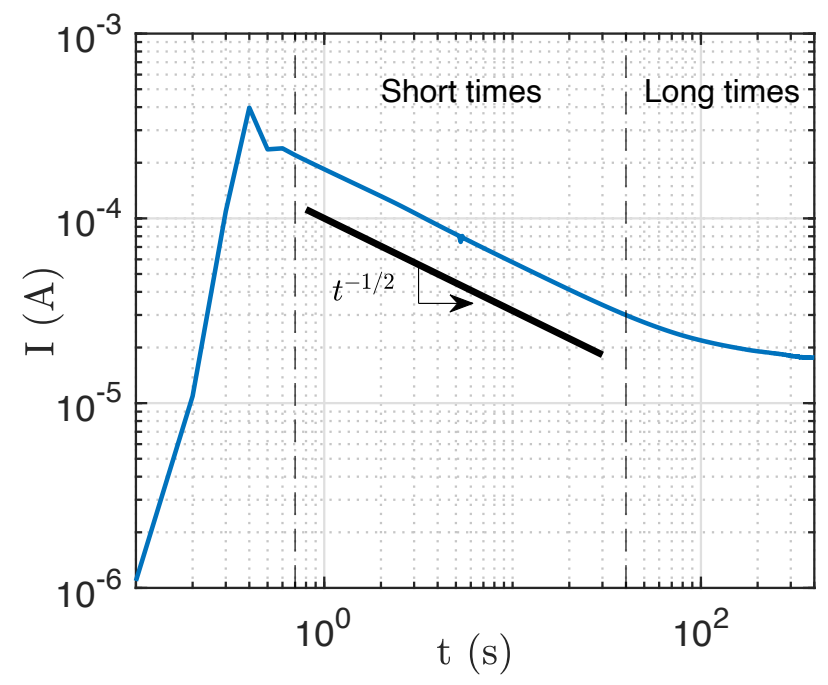

Figure 2: Typical current response of the electrochemical cell to a voltage step $\left(\log -\log\right.$ scale). A $t^{-1 / 2}$ power law is found for the scaling of the transient regime, as proposed by the Cottrell relation (Eq. 3). This experimental curve has been obtained without any acoustic forcing, which explains the longer time needed for the current to reach its plateau value $(t>400 \mathrm{~s})$.

the experimental room conditions at the time of these experiments. The reliability of the initial concentration value has also been confirmed by spectroscopy measurements regularly performed within the duration of the experiments. A similar approach has been used in [31, but the authors considered the uncertainties on the whole term $F A_{\text {eff }} \sqrt{D} C^{0}$, whereas we chose to uncorrelate the sources of discrepancies by adjusting only the value of $A_{\text {eff }}$.

The results are compiled in Table 1. We see that the effective areas $A_{\text {eff }}$ are slightly different from the nominal area $A_{\text {phy }}$ of the platinum cylinders $\left(A_{\mathrm{phy}}=7.0710^{-6} \mathrm{~m}^{2}\right)$. Due to the difficulties to seal the electrodes, there is a possibility that some varnish remains present at the border of the electrode active area in contact with the electrolytic solution or, in contrast, that the solution is slightly infiltrated around the electrode.

170 From this calibration, we deduce the value of the limit current, $I_{\text {lim,diff }}$ (also reported in Table 1, which should be observed in purely diffusive conditions [32]:

$$
I_{\lim , \operatorname{diff}}=4 F D C^{0} \sqrt{\frac{A_{\mathrm{eff}}}{\pi}}
$$

Note that different values of the constant prefactor can be found in the literature for this equation and their dependence on the configuration can be somewhat unclear [33. We then used 3D finite elements simulations of the diffusion problem to check that the value used in Eq. (4) is consistent with our geometry [29].

Table 1 also gives the experimental limit current $I_{\text {lim,0 }}$ obtained at the different electrodes without acoustic forcing. This experimental limit current $I_{\lim , 0}$ is always significantly larger than the corresponding theoretical value $I_{\text {lim,diff }}$ obtained by the calibration (by a factor between 2 and 5). As formerly underscored in numerous experimental 


\begin{tabular}{ccccccccc}
\hline Electrode & $E_{0}$ & $E_{1}$ & $E_{2}$ & $E_{3}$ & $E_{4}$ & $E_{5}$ & $E_{6}$ & $E_{7}$ \\
\hline$A_{\text {eff }}\left(\mathrm{mm}^{2}\right)$ & 6.94 & 10.88 & 8.03 & 9.27 & $\times$ & 6.68 & $\times$ & 6.16 \\
\hline$I_{\lim , \operatorname{diff}}(\mu \mathrm{A})(\mathrm{Eq} \cdot(4))$ & 3.78 & 4.74 & 4.07 & 4.38 & $\times$ & 3.71 & $\times$ & 3.57 \\
$I_{\lim , 0}(\mu \mathrm{A})$ & 10.78 & 16.02 & 10.80 & 12.34 & $\times$ & 17.14 & $\times$ & 10.93 \\
\hline
\end{tabular}

Table 1: Values of the effective area $A_{\text {eff }}$ obtained by fitting the short time response of the current

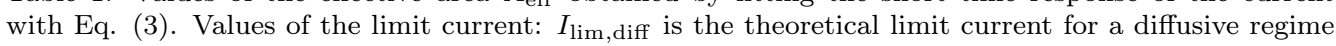
calculated with Eq. 44 and $I_{\lim , 0}$ is the experimental limit current measured without acoustic forcing at each electrode. The data for the electrodes $E_{4}$ and $E_{6}$ are not given, because the current measurements at these electrodes present bad signal to noise ratios.

studies on mass transfer, it is extremely difficult to achieve purely diffusive conditions. Uncontrolled convection was effectively present in our setup (even for smaller geometries obtained by lowering the top plate), thus enhancing the mass transfer rate as compared to diffusive conditions. Note that $I_{\mathrm{lim}, 0}$ also varies from one electrode to the other as well as from one experiment to the other. We attribute this discrepancy to the presence of low frequency fluctuations in the convection. In the following, we consequently use the theoretical value of $I_{\text {lim,diff }}$ given by Eq. (4) and computed from the fitted transient as the reference value to estimate the mass flux enhancement by the ultrasounds.

\section{Long time response of the current as a function of the acoustic forcing: a measurement of the mass flux}

190 We propose to characterize the acoustic streaming enhancement of the mass transfer by the Sherwood number defined as

$$
S h=\frac{\phi\left(P_{\mathrm{ac}}\right)}{\phi_{\mathrm{diff}}}=\frac{I_{\mathrm{lim}}\left(P_{\mathrm{ac}}\right)}{I_{\mathrm{lim}, \mathrm{diff}}},
$$

where $I_{\mathrm{lim}}\left(P_{\mathrm{ac}}\right)$ is the limit current measured under an acoustic power $P_{\mathrm{ac}}$. This Sherwood number can be interpreted as the ratio of the measured convecto-diffusive mass flux at the working electrode to the mass flux that would theoretically (Eq. (4)) occur in purely diffusive conditions at this electrode accounting for its effective area. The acoustic forcing is characterized by the acoustic Grashof number comparing the intensity of the acoustic streaming volumetric force to the viscous effects [34]:

$$
G r_{\mathrm{ac}}=\frac{32 \alpha P_{\mathrm{ac}} d_{s}}{\pi \rho c \nu^{2}},
$$

where $\rho$ is the mass density of the fluid. As already mentioned, $\alpha, d_{s}, c$, and $\nu$ are the acoustic attenuation coefficient, the diameter of the acoustic source, the sound speed, and the kinematic viscosity, respectively.

A large range of acoustic Grashof number is experimentally investigated by varying the acoustic source electric power up to $8 \mathrm{~W}$ (maximum value allowed without damaging the acoustic source). In fact, this maximum electric power corresponds to a $P_{\mathrm{ac}}=6.64$ $\mathrm{W}$ acoustic power injected in the fluid, considering the $83 \%$ efficiency of the acoustic source indicated by the manufacturer, and then to a maximum experimental value of 


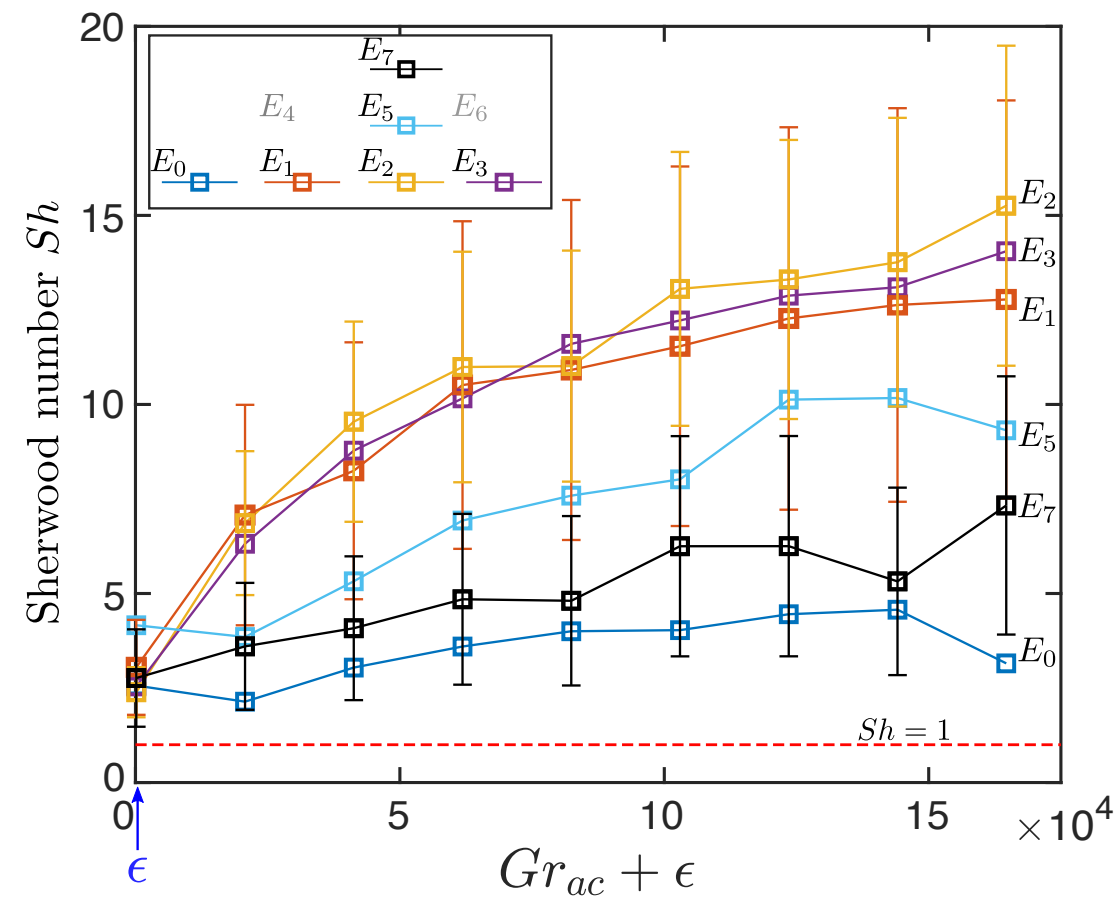

Figure 3: Sherwood number $S h$ as a function of the total Grashof number $G r=G r_{\mathrm{ac}}+\epsilon$ for six working electrodes. The offset $\epsilon$ introduced to take into account the residual convection flow observed at zero acoustic forcing is obtained by power law fits. The origin of the $x$-axis corresponds to the purely diffusive regime, never observed in the experiment due to the unavoidable convection. The first data points at $G r=\epsilon=153$ correspond to a zero acoustic forcing (effect of the convection). The other data points are obtained in presence of acoustic forcing. The vertical bars associated with the data for electrodes $E_{1}$ and $E_{7}$ denote typical uncertainties in the measurements.

the acoustic Grashof number $G r_{\mathrm{ac}}=1.64510^{5}$. In absence of acoustic forcing, Sh must theoretically be equal to 1 . However, as the influence of convection cannot be avoided, the limit $S h=1$ is never observed, even for $P_{\mathrm{ac}}=0$, i.e. $G r_{\mathrm{ac}}=0$. The presence of this residual flow has been taken into account by a typical Grashof number, $\epsilon$, which is obtained by power law fits of the experimental data at each electrode with the variable $\left(G r_{\mathrm{ac}}+\epsilon\right)$, assuming $S h=1$ (diffusive regime) at $G r_{\mathrm{ac}}+\epsilon=0$. The value of $\epsilon$ thus obtained is $\epsilon=153$, i.e. a small value compared to the experimental values of $G r_{\mathrm{ac}}$ (typically $10^{5}$ ). The experimental data expressed with the Sherwood number $S h$ are plotted as a function of the total Grashof number $G r=G r_{\mathrm{ac}}+\epsilon$ in Fig. 3. In this graph,

${ }_{215} G r=0$ corresponds to the ideal case of the diffusive regime and the first experimental data point at $G r=\epsilon$ corresponds to a zero acoustic power. For the other data points corresponding to acoustic forcing $\left(P_{\text {ac }} \neq 0\right)$, the offset $\epsilon$ due to the unmastered convection gets negligible and $G r \approx G r_{\text {ac }}$.

The evolution of the Sherwood number depends on the location of the considered 
220 probe with respect to the flow. As expected, the higher mass transfer enhancement is observed for electrodes $E_{2}$ and $E_{3}$ which are located in the middle vertical plane, in the core of the acoustic beam, and thus undergo maximum flow impact. The mass flux is here up to 15 times the expected flux in the purely diffusive regime. A factor 10 is still observed for electrode $E_{5}$ at the periphery of the beam. For the electrode $E_{1}$, with similar

225 acoustic force intensity (Fig. 1p), the Sherwood number is, however, clearly higher. An explanation could be that this electrode is in the zone influenced by the jet flow impinging on the top wall (Fig. 1 1 ). Finally, for electrodes $E_{0}$ and $E_{7}$ which are outside the beam and in weaker flow regions, the increase of the Sherwood number is initially less steep and globally weaker than for the other electrodes. The bars associated with electrodes

230 $\quad E_{1}$ and $E_{7}$ are representative of the uncertainties on the Sherwood number, which come from the temperature variations (diffusion coefficient $D$ known within $17.9 \%$ ) and the calibration procedure (effective surface $A_{\text {eff }}$ with relative error between 9 and $25 \%$ ), and are estimated through error propagation calculations using classic formula.

\section{Numerical insight into the convecto diffusive mass transfer at the wall}

235 merically investigated using the StarCCM + CFD software. The numerical modeling approach is the one used in [27]. Let us recall that this former work consisted in an experimental versus numerical comparison of the velocity field in the middle plane containing the acoustic axis for a similar configuration. The velocity fields obtained by PIV idation, no extra experimental characterization of the velocity field has been performed in the present configuration, though the inclination angle value has been changed from 34 to $27.5^{\circ}$.

As, at high Schmidt number $(S c \sim 1000)$, wall mass transfer features very fine concentration boundary layers, we have, however, adopted a slightly different mesh strategy than in [27]. Relying on the symmetry of the geometry and boundary conditions, the computational fluid domain was restricted to half of the cavity. No-slip conditions were applied at the walls and a symmetry condition was applied to the flow at the $y=0$ vertical plane containing the acoustic axis. The concentration field was modeled as a passive

250 scalar, with a zero-flux condition applied at every non active boundaries and a uniform normalized concentration imposed at the active electrodes $(0$ on the working electrodes and 1 on the counter electrode). More than one million polyhedral cells were used, with a refinement in the acoustic beam region. The wall regions were also meshed with very fine prism layers undergoing a geometric progression with the distance to the wall, in 255 order to have more than ten cells within the concentration boundary layer. The mechanical properties were set to $997.561 \mathrm{~kg} . \mathrm{m}^{-3}$ for the density and $8.88711^{-4}$ Pa.s for the dynamic viscosity, which gives a Schmidt number of 1200 if the diffusion coefficient $D$ is taken at the mid-temperature of the experimental runs.

To compute our different cases, we used the steady segregated solver implemented in StarCCM + for the lower values of the acoustic power, but the 1st order unsteady segregated solver was preferred for acoustic power greater than $2.198 \mathrm{~W}$ where unsteadiness of the velocity field occurs. The time step was typically of $0.5 \mathrm{~s}$ with at least 5 inner iterations by time steps, which has been found to insure good convergence. Note that a convergence issue was to insure passive-scalar mass conservation. The mass fluxes at 
265 the working and at the counter electrodes were thus monitored and compared during the convergence. In the steady-state calculations, it has been observed that the underrelaxation proposed for stability purpose has to be suppressed to achieve a good balance of these mass fluxes (better than $0.02 \%$ ) within a reasonable computational time. The same choice has also been done in the unsteady simulations.

$270 \quad$ The observed flow exhibits the expected y-shaped pattern already described in [27]. It is illustrated in Fig. 1 13 with a typical color-map of the velocity magnitude in the middleplane. Let us recall that the incident acoustic beam generates a first jet which impinges on the instrumented wall and flows further along it in the region of the working electrodes; the reflected acoustic beam creates a second jet going away from the instrumented wall

275 and impinging on the right-hand side wall.
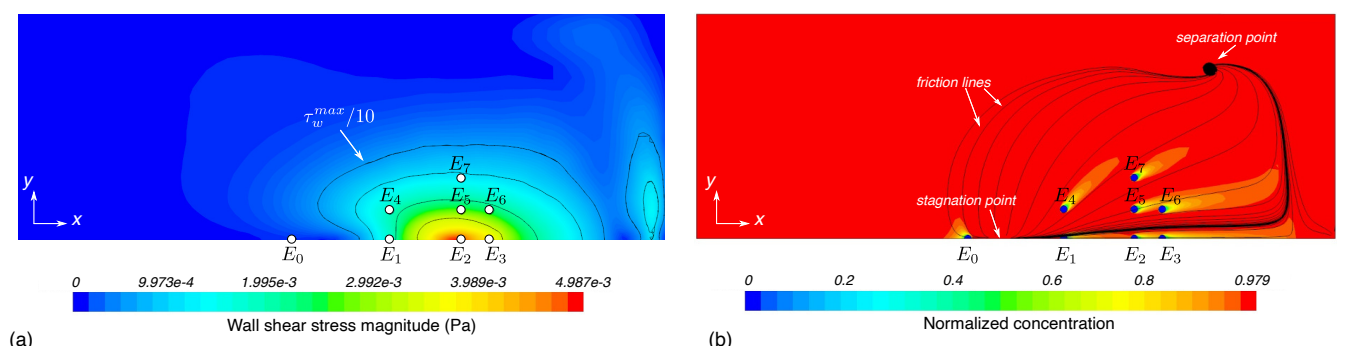

Figure 4: Wall shear stress and concentration on the instrumented wall obtained numerically at an acoustic power of $1.099 \mathrm{~W}$ with the CFD software StarCCM+. (a) Typical color-map of the wall shear stress magnitude. The electrodes are plotted as white disks. The black lines denote typical isocontours. The isocontour corresponding to one tenth of the maximum shear stress $\left(\tau_{w}^{\max } / 10\right)$ gives an idea of the zone impacted by the jet flow. (b) Typical color-map of the normalized concentration field obtained in the case where the electrodes (which appear as small blue disks) have been activated all at once, i.e. with a normalized concentration set to zero on their surface. Some friction lines are also plotted to illustrate the consistency between the shear stress at the wall and the mass transfer at the wall.

The near wall structure of the flow can be observed in Fig. 4a, which shows a typical color-map of the wall shear stress magnitude on the instrumented wall $\left(P_{\mathrm{ac}}=1.099\right.$ $\mathrm{W})$. Two high-shear zones are observed. The more intense zone corresponds to the friction region of the main jet, which flows around the symmetry plane in the $x$ direction

280 (rightward on the plot). The isocontour corresponding to one tenth of the maximum shear stress $\left(\tau_{w}^{\max } / 10\right)$ gives an idea of this friction region. Other isocontours at $\tau_{w}^{\max } / 1.5$, $\tau_{w}^{\max } / 2.5$, and $\tau_{w}^{\max } / 5$ comprise successively the electrodes $E_{2}$ and $E_{3}$, then the electrodes $E_{5}$ and $E_{6}$, and finally the electrodes $E_{1}, E_{4}$ and $E_{7}$. The clearly less intense second zone corresponds to the flow forced by the presence of the lateral wall, moving along this

285 wall away from the symmetry plane (upward on the plot). Finally, on the left of the stagnation point (see Fig. $4 \mathrm{~b}$ ), zone where the $E_{0}$ electrode is located, the fluid flows in the $-x$ direction (leftward on the plot). It has to be noted that the shear stress contours in the impact area resembles the isovalues of the acoustic force (Fig. 1 b), but with a less symmetric shape.

The color-map of the normalized concentration is plotted in Fig. 4 b together with some friction lines (streamlines of the wall shear stress, colored in grey) for the same case at $P_{\mathrm{ac}}=1.099 \mathrm{~W}$. The electrodes, which appear as small blue disks, have been activated all at once, i.e. with a normalized concentration set to zero on their surface, 
to obtain this figure. We see that most of the friction lines originate at the stagnation

point of the jet driven by the incident acoustic beam (located in the symmetry plane between $E_{0}$ and $E_{1}$ ) and terminate at another characteristic point, which appears as a stagnation point, away from the symmetry plane. Even for this relatively low forcing, the concentration plume at each electrode appears to be clearly driven by the convection and strongly correlated with the friction lines. This illustrates the consistency between

зоо mass transport and near wall hydrodynamics. Note that the $E_{3}$ and $E_{6}$ electrodes are in the wake of two of their counterparts, the $E_{2}$ and $E_{5}$ electrodes, respectively, so that the mass transfer at these electrodes has to be computed after having deactivated all the other electrodes, in order to mimic the experimental conditions in which each electrode is used alone.

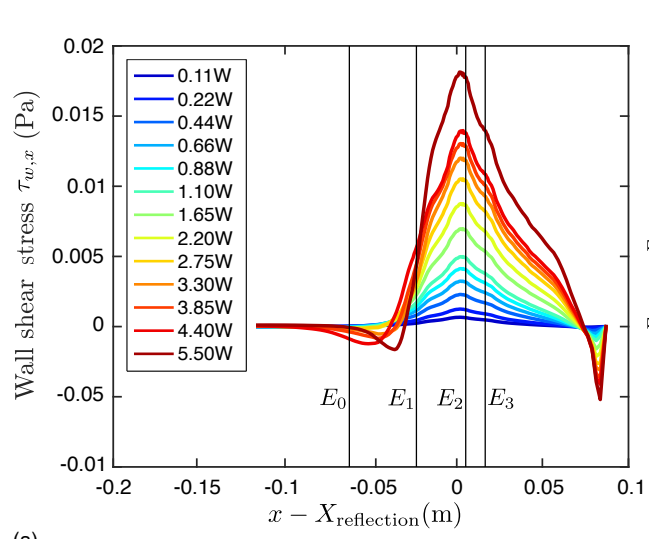

(a)

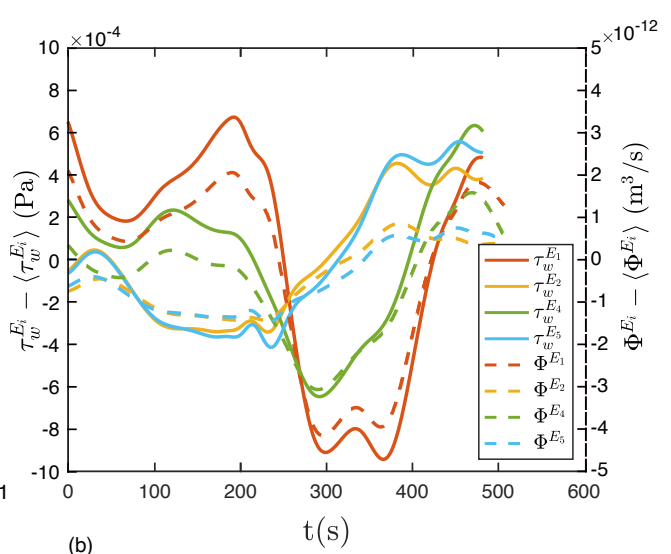

(b)

Figure 5: (a) Longitudinal profiles of the $x$-component of the wall shear stress $\tau_{w, x}$ along the symmetry axis containing electrodes $E_{0}, E_{1}, E_{2}$, and $E_{3}$ for many different values of the acoustic power $P_{\text {ac }}$. $\tau_{w, x}$ is expressed as a function of $x-X_{\text {reflection, where } X_{\text {reflection }} \text { is the abcissa of the intersection point }}$ between the acoustic axis and the instrumented plane. The maximum value of $\tau_{w, x}$ increases with $P_{\text {ac }}$ and is close to $x=X_{\text {reflection. }}$ (b) Fluctuations of the wall shear stress $\tau_{w}^{E_{i}}$ (solid lines, left-hand side axis) and mass flux $\phi^{E_{i}}$ (dashed lines, right-hand side axis) over $500 \mathrm{~s}$ at electrodes $E_{1}, E_{2}, E_{4}$, and $E_{5}$ for an acoustic power $P_{\mathrm{ac}}=5.495 \mathrm{~W}$. The time-averaged values have been subtracted for the sake of clarity. The mass flux appears to follow the fluctuations of the flow without phase shift.

In order to see how the wall shear stress evolves with the acoustic power $P_{\mathrm{ac}}$, figure $5 \mathrm{a}$ shows the instantaneous longitudinal component of the wall shear stress, $\tau_{w, x}$, along the symmetry axis, for several acoustic powers. We see that the maximum shear increases with the acoustic power, which induces the development of steep gradients on both sides of this maximum value. Note that the maximum shear location does not depend on the

310 applied acoustic power and is very close to $x=X_{\text {reflection, }}$ i.e. to the point where the acoustic axis crosses the instrumented plane. In contrast, the position of the stagnation point where the wall shear stress goes to zero, upstream of the reflection point, varies with the acoustic power. The negative values of $\tau_{w, x}$ downstream of the reflection point, next to $x-X_{\text {reflection }}=0.08 \mathrm{~m}$, correspond to the recirculation cell due to the nearby end wall. The position of the electrodes along the symmetry axis is indicated as thin black lines in the figure. As expected, the electrode $E_{0}$ is outside the forcing area and is 
submitted to low and negative values of shear stress. Electrodes $E_{1}$ and $E_{3}$ are located on both sides of the maximum shear stress and electrode $E_{2}$ is very close to this maximum shear stress.

For acoustic powers greater than $2.198 \mathrm{~W}$, the flow obtained in the numerical simulations appears to be unsteady. No purely periodic regime has been observed with the considered values of the parameters; the flow variations appear rather as low frequency random variations. This type of dynamics has already been observed in some of our former works [16, 35, 36]. In [36] in particular, we have shown the very rich and sensitive dynamics in such acoustically driven flows, with a flow regime which could change from periodic to quasiperiodic and chaotic within a $15 \%$ wide acoustic power range.

In the following, in order to be able to compare with the experimental data which are obtained by measurements at the electrodes, we will compute values averaged over the electrodes surfaces. These values will be denoted with a superscript $E_{i}$ to refer to the corresponding electrode, e.g. $\tau_{w}^{E_{1}}$ and $\phi^{E_{1}}$ for the wall shear stress and the mass flux at the electrode $E_{1}$, respectively.

The unsteady behavior observed in the simulations for the larger values of the acoustic power is illustrated in Fig. 5b by the plot of the time fluctuations of the wall shear stress (solid lines, scale on the left-hand side axis) and of the wall mass flux (dashed lines,

335 scale on the right-hand side axis) at some representative electrodes. To obtain these data, the time averaged values of wall shear stress $\left\langle\tau_{w}^{E_{i}}\right\rangle$ and mass flux $\left\langle\phi^{E_{i}}\right\rangle$ at the electrodes have been subtracted to the instantaneous values $\tau_{w}^{E_{i}}$ and $\phi^{E_{i}}$, respectively. The figure first shows clearly that simulations on very long times $(t \gg 500 \mathrm{~s})$ would be necessary to converge statistical quantities in such acoustic streaming configuration.

340 The plot of both shear stress and mass flux together on the same graph allows to see that these two quantities are very well correlated for all the electrodes, with almost no phase shift, indicating that for large Schmidt numbers $(S c \sim 1000)$, the concentration field at the electrodes is controled by the wall shear stress. At electrodes $E_{2}$ and $E_{5}$, which are aligned at the same $x$ coordinate on the instrumented plate, downstream of the

345 reflection point, fluctuations with similar variations (amplitude and phase) are observed. The fluctuations on electrodes $E_{1}$ and $E_{4}$, aligned at the same $x$ coordinate, but upstream of the reflection point, are also in phase, but anti-correlated with those observed at the downstream electrodes $E_{2}$ and $E_{5}$. Their amplitude is rather larger, particularly at electrode $E_{1}$. This is consistent with what was observed in [36] in a multi-reflection

350 situation, where the fluctuations principally occur in the corners between the jets and the corresponding impacted wall, upstream of the reflection point.

\section{Analysis of the results at the electrodes}

\subsection{Wall shear stress at the electrodes}

To quantify the wall shear stress computed at the electrodes, it is interesting to determine a reference characteristic value. An order of magnitude of the shear stress at the upper wall in the impingement area can be estimated by the momentum accumulated all along the path of the fluid particles in the incident jet. The momentum versus wall friction balance projected on the $x$ direction (tangent to the instrumented wall) can be written as:

$$
\tau_{w}^{O M} \approx \frac{2 \alpha P_{\mathrm{ac}} L_{\mathrm{ac}} \cos \theta \sin \theta}{\frac{\pi d_{s}^{2}}{4} c}
$$


where $L_{\mathrm{ac}}=195 \mathrm{~mm}$ is the length of the acoustic path from the entrance wall to the instrumented wall (see Fig. 1 1 ) and the factors $\cos \theta$ and $\sin \theta$ come from the projections on the $x$ direction of the incident jet momentum and of the estimated jet cross section $\frac{\pi d_{s}^{2}}{4}$, respectively. This order of magnitude expression $\tau_{w}^{O M}$ can be adjusted with a multiplicative factor to fit the initial variation with $P_{\mathrm{ac}}$ of the shear stress computed at electrode $E_{2}$, which is the most influenced by the flow. We observe that a factor $1 / 4$ gives a reasonable fit and then define the reference shear stress $\tau_{w}^{0}$ as:

$$
\tau_{w}^{0}=\frac{1}{4} \tau_{w}^{O M}=\frac{0.5 \alpha P_{\mathrm{ac}} L_{\mathrm{ac}} \cos \theta \sin \theta}{\frac{\pi d_{s}^{2}}{4} c} .
$$

A striking feature of this estimate is its increase with increasing acoustic path $L_{\mathrm{ac}}$. Of course, this cannot be extrapolated to very long distances and is expected to hold as long as the acoustic path stays smaller than the acoustic attenuation length, i.e. $\alpha L_{\mathrm{ac}} \ll 1$ (here $\alpha L_{\mathrm{ac}} \approx 210^{-2}$ ).
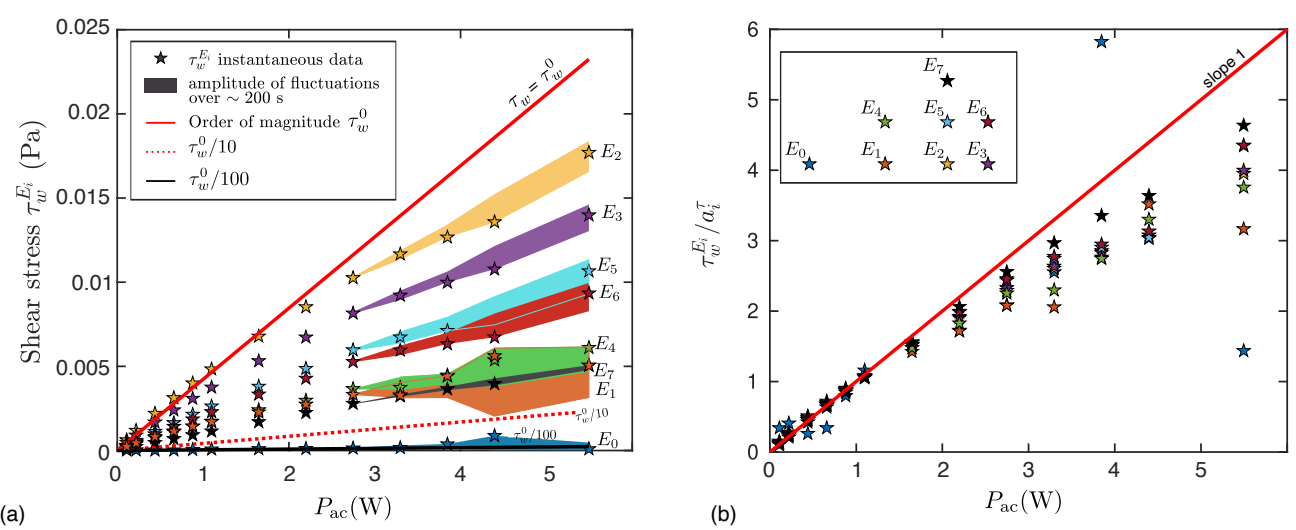

Figure 6: (a) Numerical data of the shear stress at the electrodes, $\tau_{w}^{E_{i}}$, as a function of the acoustic power. The colored regions correspond to the values of $\tau_{w}^{E_{i}}$ explored in the unsteady cases. The stars indicate steady or instantaneous values. The solid red line is a linear approximation of the shear stress, $\tau_{w}^{0}$ (Eq. (8)), coming from an order of magnitude approximation (Eq. 77). The dashed red line and the solid black line are lines of equation $\tau_{w}=\tau_{w}^{0} / 10$ and $\tau_{w}=\tau_{w}^{0} / 100$, respectively. All data, except for the electrode $E_{0}$, are in the range between $\tau_{w}^{0}$ and $\tau_{w}^{0} / 10$. (b) The same data after normalization of $\tau_{w}^{E_{i}}$ by the coefficient $a_{i}^{\tau}$ obtained by a least squares fit of the data at each electrode with a power law behavior $\tau_{w}^{E_{i}}=a_{i}^{\tau} P_{\mathrm{ac}}^{\beta_{i}}$. Except for $E_{0}$, all data collapse well on a close to linear behavior (solid red line) for small acoustic forcing, whereas for higher forcing, they exhibit different variations with smaller slopes. The exponents $\beta_{i}$ are given in Table 2 The code of the colors used for the different electrodes is given as inset in (b).

Figure 6 a displays the numerically computed values of the shear stress at the electrodes, $\tau_{w}^{E_{i}}$, as a function of the acoustic power $P_{\mathrm{ac}}$ : the colored zones correspond to the values explored by the unsteady solutions over at least $200 \mathrm{~s}$; the stars correspond to the steady values or to the instantaneous values obtained at the end time of the unsteady simulations, values which are also used in the following plots. The reference value of the 
shear stress $\tau_{w}^{0}$ is plotted as a solid red line. As expected, at low values of the acoustic power $P_{\text {ac }}$, this line fits fairly well the values observed at the $E_{2}$ electrode, the nearest to the maximum shear location. Then, depending on its location in the high shear region, each electrode exhibits a more or less steep increase in shear stress with the acoustic power. The dotted red line corresponds to $\tau_{w}^{0} / 10$; we see that most of the electrodes are located in an area where the shear stress is in the range $\left[\tau_{w}^{0} / 10, \tau_{w}^{0}\right]$. It is thus worth defining the jet friction zone as the zone where the wall shear stress value is within this range. The values at electrode $E_{0}$ are below this arbitrary limit; actually the shear stress at this electrode is rather on the order of $\tau_{w}^{0} / 100$, as illustrated by the black line in the figure. This is not surprising since $E_{0}$ is effectively not in the same flow area as the other electrodes, as already seen in Fig. 4. Note also that the increase in wall shear stress for a given electrode is not linear: the observed slope clearly decreases at higher acoustic power. The shear stress rather follows a power law which can be written as:

$$
\tau_{w}^{E_{i}}=a_{i}^{\tau} P_{\mathrm{ac}}^{\beta_{i}}
$$

with the exponent $\beta_{i}$ and the coefficient $a_{i}^{\tau}$ depending on the electrode (subscript $i$ ). To

390 better illustrate the non linear behavior of the shear stress at the electrodes, a least-square fit has been realized to get the coefficient $a_{i}^{\tau}$ and the exponent $\beta_{i}$ for each electrode. The $\tau_{w} / a_{i}^{\tau}$ curves are shown as a function of $P_{\text {ac }}$ in Fig. 6p. We see that below an acoustic power of $2 \mathrm{~W}$, all electrodes data, except for $E_{0}$, collapse on the same curve with a close to linear behavior (solid red line). The values of the exponent $\beta_{i}$ for each electrode are given in Table 2. As expected from Fig. 6b, all the values are below 1. However, there is not any clear tendency for the variation of $\beta_{i}$ with the electrodes. On the symmetry axis, the strongest exponents $\beta_{i}$ are obtained for the electrodes $E_{2}$ and $E_{3}$ which are the closest to the reflection point. Strongest values of $\beta_{i}$, however, are obtained for electrodes that are away from this axis. \\ 6.2. Mass flux at the electrodes}

In this section, we will analyze the enhancement of the wall mass transfer at the electrodes and, for that, will compare the numerical and experimental results and study their connections with the numerically obtained shear stress. Note first that the experimental approach presented in section 4 was directly used to measure wall mass transfer, but it was inspired by the polarometry technique where the current measurement is used as an indirect measurement of the wall shear rate [37, 38, 39]. Indeed, in this technique, the wall shear rate $\tau_{w} / \mu$ is deduced from the measured current $I_{\text {lim }}$ using the well known Leveque equation

$$
I_{\lim }=k_{\text {Lev }}\left(\frac{\tau_{w}}{\mu}\right)^{1 / 3}
$$

where the constant $k_{\text {Lev }}$ and even the exact value of the exponent are generally obtained after a proper in situ calibration. Nevertheless, a theoretical expression of the Leveque coefficient has been derived for circular electrodes [39]:

$$
k_{\text {Lev }}=0.686 A^{5 / 6} F C^{0} D^{2 / 3} \pi^{1 / 6},
$$

expression we will use in the following. It can be noted that Eq. 10 is only valid in the large Schmidt number limit (i.e. when the thickness of the concentration boundary 


\begin{tabular}{ccccccccc}
\hline Electrode $E_{i}$ & $E_{0}$ & $E_{1}$ & $E_{2}$ & $E_{3}$ & $E_{4}$ & $E_{5}$ & $E_{6}$ & $E_{7}$ \\
\hline$\beta_{i}$ & 0.814 & 0.752 & 0.843 & 0.858 & 0.852 & 0.898 & 0.910 & 0.971 \\
\hline$\gamma_{i}^{\text {num }}$ & 0.669 & 0.774 & 0.839 & 0.839 & 0.863 & 0.877 & 0.875 & 0.945 \\
\hline$\gamma_{i}^{\exp }$ & 0.54 & 0.93 & 1.17 & 1.14 & $\times$ & 1.20 & $\times$ & 0.90 \\
\hline
\end{tabular}

Table 2: Characteristic exponents of scaling laws concerning the shear stress $\tau_{w}^{E_{i}}$ or the mass transfer (represented by the Sherwood number $S h$ ) at the electrodes as a function of the acoustic Grashof number $G r_{\text {ac }} \cdot \beta_{i}$ is the scaling found numerically for the shear stress (Eq. 99). $\beta_{i} / 3$ is also the scaling for the mass transfer if we assume the validity of the Leveque formula 10 . Finally, $\gamma_{i}^{\text {num }} / 3$ and $\gamma_{i}^{\exp } / 3$ are the scalings for the mass transfer obtained directly from the numerical and experimental data, respectively. Note that an exponent $1 / 3$ is obtained for the mass transfer in the ideal case of the Leveque law validity and a shear stress proportional to the acoustic power, so that $\beta_{i}, \gamma_{i}^{\text {num }}$, and $\gamma_{i}^{\text {exp }}$ have to be compared with 1 .

layer is much smaller than the thickness of the hydrodynamics boundary layer) and for 415 (fificiently high convective flow: indeed, it predicts a zero limit current at zero shear rate, whereas the limit current is known to tend towards a non zero value, $I_{\text {lim,diff }}$, corresponding to purely diffusive conditions.

From the Leveque formula 10 , we can express the Sherwood number $S h=I_{\lim } / I_{\text {lim,diff }}$ as a function of the wall friction coefficient defined as $C_{f}=\tau_{w} / \tau_{w}^{0}$ and of the acoustic

Grashof number $G r_{\text {ac }}$. We obtain a dimensionless form of the Leveque formula:

$$
S h=a S c^{\frac{1}{3}} C_{f}^{\frac{1}{3}} G r_{\mathrm{ac}}^{\frac{1}{3}},
$$

with $a=\frac{0.686 \pi^{2 / 3}}{4^{5 / 3}} \frac{\left(A_{\mathrm{phy}} L_{\mathrm{ac}}\right)^{1 / 3}}{d_{s}}(\cos \theta \sin \theta)^{1 / 3}$, which will be confronted with our data.

We will first use our numerical and experimental data to test the connection between the mass transfer at the wall and the wall shear stress, as proposed by the Leveque formula. Refering to Eq. $\sqrt{12}$, we then express $S h$ as a function of $C_{f} G r_{\text {ac }}$ for all our data in Fig. 7 a. A log-log scale is chosen to better see the characteristic exponents of these variations.

The numerical data corresponding to all the electrodes and all the forcing values are plotted as stars, with colors depending on the considered electrode (the code of the colors is given as inset in Fig. 7b). We see that all the numerical data collapse on a master curve over the major part of the parameters range and this master curve nearly corresponds to the solid black line given by the dimensionless Leveque formula (12) without any adjustable parameter. Leveque formula then remarkably holds within our forcing parameters range, though it should be more suitable for turbulent flows. Note that the slope is globally slightly smaller than the $1 / 3$ exponent given by Eq. 12 at the moderate and large values of $C_{f} G r_{\mathrm{ac}}$ and that, for $C_{f} G r_{\mathrm{ac}}<210^{2}$, the numerical data seem to leave the characteristic law with a far smaller slope, observation which could be attributed to a too small convective transport for the Leveque formula to remain valid.

The experimental data are also plotted in Fig. 7 a where they appear as squares, with the same code of colors to distinguish the electrodes. As no direct measurement of the shear stress is available, we use the values obtained numerically for the considered acoustic power to calculate the abscissa for each experimental data point. Typical error bars are also plotted, but only for two selected electrodes $E_{1}$ and $E_{7}$ for the sake of 

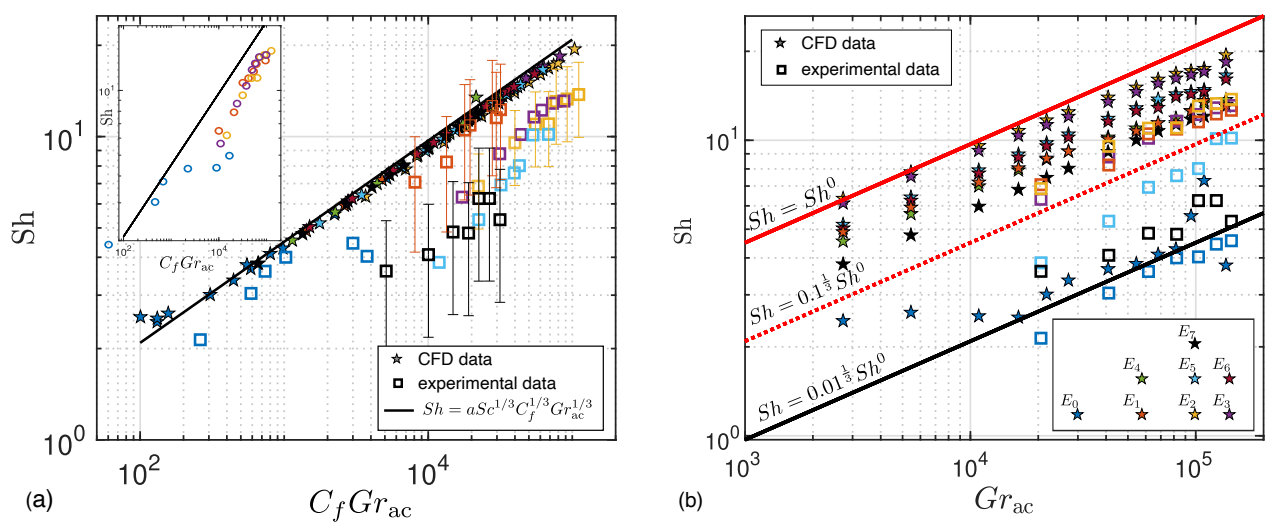

Figure 7: (a) Dimensionless mass transfer given by the Sherwood number $S h$ as a function of the wall shear stress expressed by the dimensionless product $C_{f} G r_{\mathrm{ac}}$. Stars are used for the numerical data and squares for the experimental data. The solid black line gives the Leveque formula in its dimensionless form (see Eq. 12 ). (b) For the same data, Sherwood number $S h$ now expressed as a function of the acoustic power through the acoustic Grashof number $G r_{\text {ac }}$. Some characteristic lines are plotted using Eq. 12 with a shear stress based on the estimated reference shear stress $\tau_{w}^{0}$ (Eq. (8)): the solid red line $S h=S h^{0}$ for $\tau_{w}=\tau_{w}^{0}\left(C_{f}=1\right)$, the dashed red line $S h=0.1^{1 / 3} S h^{0}$ for $\tau_{w}=\tau_{w}^{0} / 10\left(C_{f}=0.1\right)$ and the solid black line $S h=0.01^{1 / 3} S h^{0}$ for $\tau_{w}=\tau_{w}^{0} / 100\left(C_{f}=0.01\right)$. The code of the colors used for the different electrodes is given as inset in (b).

clarity. Although the overall figure exhibits a certain consistency, the experimental data appear to be significantly lower than the expected dimensionless Leveque law. They also

445 do not collapse on any single master curve and their variations with $C_{f} G r_{\text {ac }}$ seem to correspond to exponents larger than the value $1 / 3$ expected from 12 .

Note that there is an uncertainty on the position of the reflection point on the instrumented plane, which has not been accounted for in this plot. A deeper analysis concerning $E_{0}, E_{1}, E_{2}$, and $E_{3}$, the electrodes on the symmetry axis, i.e. at $y=0$, shows that assuming a 5 to $6 \mathrm{~mm}$ shift of the reflection point in the $-x$ direction would lead to fairly aligned data points on a single curve. Indeed, in this case, $E_{1}$ would undergo higher shear, while $E_{2}$ and $E_{3}$ would undergo lower shear (see Fig. 5 a). This is illustrated in the inset of Fig. $7 \mathrm{a}$, where the corrected experimental data points for the three electrodes $E_{1}$ to $E_{3}$ in the friction zone gather on a single curve. This curve, however, remains below the theoretical Leveque law and with a slightly higher exponent.

The fact that the experimental data points are systematically below the Leveque curve is more difficult to explain from uncertainty considerations. One likely explanation is that the acoustic power transmitted to the fluid in the experiment was smaller than in our estimation. Let us recall that we estimated it from the input electric power measured with an inline wattmeter and a global $83 \%$ efficiency provided by the supplier at the delivery of the acoustic source. In fact, the source was not new and we cannot exclude that its efficiency was significantly smaller than its initial value.

In Fig. 7a, the plots have mixed the mass transfer experimental data with the numerical shear stress. In order to have plots in which the representation of the experimental data points does not depend on the numerical simulations, we can rather express the 
mass transfer as a function of the acoustic power, i.e. the Sherwood number $S h$ as a function of the acoustic Grashof number $G r_{\mathrm{ac}}$. Such plots of $S h$ versus $G r_{\mathrm{ac}}$ are shown in Fig. $7 \mathrm{~b}$ for both the experimental and numerical data. Some characteristic lines are also plotted in the figure. They are obtained from the dimensionless Leveque law (12) expressed for specific constant values of the friction coefficient $C_{f}$, i.e. for shear stress proportional to the reference shear stress $\tau_{w}^{0}$. Knowing from the numerical data that the shear stress in the jet friction zone is in the range $\left[\tau_{w}^{0} / 10, \tau_{w}^{0}\right]$ (Fig. 6a), $C_{f}$ will be in the $[0.1,1]$ range. Substituting $C_{f}=1 \mathrm{in} \mathrm{Eq.} 12$ yields the following reference value for the Sherwood number:

$$
S h^{0}=a S c^{\frac{1}{3}} G r_{\mathrm{ac}}^{\frac{1}{3}},
$$

475 and the Sherwood number $S h$ in the jet friction zone is then expected to be in the range $\left[0.1^{1 / 3} S h^{0}, S h^{0}\right]$. This is illustrated in Fig. $7 \mathrm{~b}$ where all the numerical data (except those for the electrode $E_{0}$ ) fall within this range, i.e. between the solid and dotted red lines. Note that $0.1^{1 / 3}=0.464 \approx 1 / 2$ : this indicates that, according to the numerical data, the mass transfer in the whole jet friction zone is within about a factor $1 / 2$ with respect to

480 its maximum value at the reflection point. Concerning the experimental data, they are still below the numerical data, and the Sherwood number in the friction zone is rather above the solid black line corresponding to $0.01^{1 / 3} S h^{0} \approx S h^{0} / 5$.

We can now consider the change of the Sherwood number with the acoustic Grashof number for the different electrodes. Note first that the characteristic lines $S h \propto S h^{0}$ give

485 a $1 / 3$ exponent, which is based on the Leveque law 10 and on the fact that they assume that $\tau_{w} \propto \tau_{w}^{0}$, i.e. the shear stress is proportional to the acoustic power (Eq. (8)). As the numerical results have shown that such property of the shear stress is only valid for low acoustic forcing (see Fig. 6b), it explains why, for the numerical data, the exponent is only close to $1 / 3$ in this limit and decreases for larger forcings. The fact that the 490 exponent systematically appears as slightly smaller than $1 / 3$, even at low forcings, can be attributed to the observed slope in Fig. 7 a slightly smaller than $1 / 3$, i.e. to a slight departure from the Leveque law. In contrast, the exponent for the experimental data appear to be larger than $1 / 3$. More precise characteristic exponents expressed as $\gamma_{i} / 3$ can be obtained by least squares fits of the form $S h \propto G r_{\mathrm{ac}}^{\gamma_{i} / 3}$ for the numerical data and

$495 S h-1 \propto\left(G r_{\mathrm{ac}}+\epsilon\right)^{\gamma_{i} / 3}$ for the experimental data, and the values of $\gamma_{i}$ thus obtained will be denoted as $\gamma_{i}^{\text {num }}$ and $\gamma_{i}^{\text {exp }}$, respectively. Another scaling of the Sherwood number can be obtained from the numerical data if we assume the validity of the Leveque formula (10): in that case, we get an exponent $\beta_{i} / 3$, where $\beta_{i}$ is the scaling found previously for the shear stress (Eq. (9)).

soo Table 2 gives the values of $\beta_{i}$ and $\gamma_{i}$ at the different electrodes, to be compared with the corresponding value 1 obtained in the reference case $S h \propto S h^{0}$. As shown in the previous subsection, the $\beta_{i}$ values obtained from the fit of the numerical shear stress are smaller than 1 , in the range [0.75, 0.97]. The values of $\gamma_{i}^{\text {num }}$, directly obtained from the fit of the numerical mass transfer, are very close to the values of $\beta_{i}$, except for the electrode

${ }_{505} E_{0}$ which is outside the jet friction zone. This still indicates that the departure from the Leveque law remains small for the numerical data. From these numerical results, a typical global exponent for the $S h$ versus $G r_{\text {ac }}$ scaling law in the jet friction zone is $\gamma^{\text {num }} / 3 \sim 0.85 / 3$. In contrast, the values of $\gamma_{i}^{\exp }$ obtained from the fit of the mass transfer experimental data are quite different, higher than the numerical values and, for some electrodes, even higher than 1 . This is, however, consistent with what was observed 
previously in Fig. 7 a.

\section{Conclusion}

The present study is based on an electrochemical approach to estimate wall mass transfer enhancement by acoustic streaming. The hydrodynamic configuration is very

${ }_{515}$ similar to that presented in a former paper focused on the hydrodynamics effects [27]: a 2 $\mathrm{MHz}$ ultrasound beam is directed towards a wall with an angle $\left(27.5^{\circ}\right.$ here $)$ so as to create an impinging acoustic streaming jet after a nearly $200 \mathrm{~mm}$ acoustic path; the reflection of the beam on the wall creates a second jet providing a so-called y-shape to this jet flow. The current measured at the working electrodes, implemented in the friction zone

520 of the jet, allows to show that the wall mass flux is enhanced by a factor greater than ten over the several $\mathrm{cm}^{2}$ of the investigated area, with acoustic powers of a few watts only (see Fig. 3). This contrasts with former electrochemistry works using ultrasounds in the $\mathrm{kHz}$ range, with powers of hundreds of watts delivered at small distances from the target [24, 25]. Our analysis partly relies on a calibration procedure based on the short

${ }_{525}$ time response of the current, following Cottrell analysis [30. This calibration enables us to get rid of an experimental bias associated with a difficultly mastered convection and therefore to compute the flux in the ideal case of a diffusive regime. Though significant at low forcing, this convection is shown to be negligible compared with acoustic streaming flows for most of the investigated forcings.

530 An order of magnitude model is proposed to link the wall mass transfer to the input acoustic power. It is based on a momentum budget to determine a characteristic wall shear stress, $\tau_{w}^{0}$, proportional to the acoustic power, from which the wall mass transfer is deduced using the well-known Leveque formula. The model thus yields a typical Sherwood number characterizing the wall mass transfer, $S h^{0}$, as a function of the acous-

535 tic Grashof number $G r_{\mathrm{ac}}$ characterizing the acoustic forcing and the Schmidt number characterizing the transport properties of the considered solution, both involved with a $1 / 3$ power. Note that the characteristic wall shear stress $\tau_{w}^{0}$ is also found to increase proportionally to the length $L_{\mathrm{ac}}$ over which the acoustic beam forces the jet before it impinges on the wall. In the considered parameters range, for which $L_{\mathrm{ac}}$ is far smaller

540 than the typical acoustic attenuation length $1 / \alpha$, the action on the wall mass transfer is thus expected to be all the more efficient as the source is far from the wall.

A numerical model of the experiment is also implemented using the commercial CFD software StarCCM $+{ }^{\circledR}$, coupled with a homemade linear acoustic model solved under Matlab ${ }^{\circledR}$ giving the driving acoustic force. The numerical model gives access to the near wall hydrodynamics (in particular the wall shear stress) together with the wall mass flux obtained through a passive scalar approach. An important output of this modeling is the very good agreement of the results with the Leveque formula. Concerning hydrodynamics, the maximum wall shear stress location is observed to be independent of the applied acoustic power and to coincide with the reflection point of the acoustic beam axis. This maximum value can be approximated at moderate forcing by the characteristic wall shear stress $\tau_{w}^{0}$, while it evolves more slowly than $\tau_{w}^{0}$ at higher forcings. A friction zone area is then formally defined around this point as the zone over which the same order of magnitude holds, that is to say the zone with a wall shear stress larger than one tenth of $\tau_{w}^{0}$. The order of magnitude approach predicts that the Sherwood number in this region should evolve with the acoustic Grashof number with a $1 / 3$ power, with 
values between approximately $S h^{0}$ and $S h^{0} / 2$, depending on the considered location more or less close to the reflection point. The numerically obtained Sherwood number is in very fair agreement with this prediction, though its increase with $G r_{\mathrm{ac}}$ is less steep than expected, featuring typically a power $0.85 / 3$ instead of $1 / 3$.

The experimentally measured wall mass flux values appear to be systematically smaller than those obtained from the numerical approach, and this could be the consequence of less acoustic energy injected due to uncontrolled power losses at the level of the transducer. The proposed order of magnitude is still interesting since the data from the electrodes located in the friction zone give a Sherwood number greater than about

${ }_{565} S h^{0} / 5$. Moreover, despite some experimental dispersion, the increase of the Sherwood number with the acoustic Grashof number exhibits a consistent global behavior (see Figs. $7 \mathrm{a}$ and $\mathrm{b})$.

To investigate the influence of the Schmidt number, it can be useful to take advantage of the analogy between transport of concentration and transport of temperature. As

570 the Prandtl number $\operatorname{Pr}=\frac{\nu}{\kappa}$ can easily take values around 1 for aqueous solutions, an analogous study based on heat transfer could give information on the solute transfer in solutions with smaller Schmidt numbers $(S c \approx 1)$.

Finally, it must be noted that the investigated experimental conditions, namely acoustic powers from 0 to $6.64 \mathrm{~W}$ delivered by a transducer at $2 \mathrm{MHz}$, correspond to steady

575 laminar to low frequency chaotic flow regimes. Increasing the forcing intensity should induce a turbulent regime and different scaling laws could then be observed. In the future, the electrochemical technique implemented in the present paper could be used to assess segregation improvement in real crystal growth configurations through an experimental modeling in ambient temperature experiments. The investigation of unsteady regimes of acoustic streaming flows in liquids could also benefit from the use of this technique.

\section{Acknowledgements}

This collaborative work was supported by the PHC Maghreb Partnership Program no. 36951NG. The funding was obtained through the Carnot Institute Ingenierie@Lyon. The support from the PMCS2I of Ecole Centrale de Lyon for the numerical calculations and from Meca3D (Insa Lyon) for the experiment are also gratefully acknowledged. The authors wish to thank Pr. A. Bouabdallah and F. Lusseyran for fruitful discussions on polarography techniques and P. Vilanoba for his preliminary work on numerical simulations.

\section{References}

[1] J. Klima, Application of ultrasound in electrochemistry. An overview of mechanisms and design of experimental arrangement, Ultrasonics 2 (2011) 202-209.

[2] W. Dridi, D. Henry, H. Ben Hadid, Influence of acoustic streaming on the stability of melt flows in horizontal Bridgman configurations, Journal of Crystal Growth 310 (2008) 1546-1551.

[3] P. Jarry, M. Rappaz, Recent advances in the metallurgy of aluminium alloys. Part I: Solidification and casting, Comptes Rendus Physique 19 (2018) 672-687.

[4] G. S. B. Lebon, G. Salloum-Abou-Jaoude, D. Eskin, I. Tzanakis, K. Pericleous, P. Jarry, Numerical modelling of acoustic streaming during the ultrasonic melt treatment of direct-chill (DC) casting, Ultrasonics Sonochemistry 54 (2019) 171-182.

[5] J.-P. Garandet, N. Kaupp, D. Pelletier, The effect of lid driven convective transport on lateral solute segregation in the vicinity of a crucible wall, Journal of Crystal Growth 361 (2012) 195-200. 
[6] M. Chatelain, S. Rhouzlane, V. Botton, M. Albaric, D. Henry, S. Millet, D. Pelletier, J.-P. Garandet, Towards wall functions for the prediction of solute segregation in plane front directional solidification, Journal of Crystal Growth 475 (2017) 55-69.

[7] G. N. Kozhemyakin, Influence of ultrasonic vibrations on the growth of semiconductor single crystals, Ultrasonics 35 (1998) 599-604.

[8] M. Barthès, G. Mazue, D. Bonnet, R. Viennet, J.-Y. Hihn, Y. Bailly, Characterization of the activity of ultrasound emitted in a perpendicular liquid flow using Particle Image Velocimetry (PIV) and electrochemical mass transfer measurements, Ultrasonics 59 (2015) 72-78.

[9] V. Frenkel, R. Gurka, A. Liberzon, U. Shavit, E. Kimmel, Preliminary investigations of ultrasound induced acoustic streaming using particle image velocimetry, Ultrasonics 39 (2001) 153-156.

[10] M. Dhahbi, M. Ben Chiekh, B. Gilles, J.-C. Béra, A. Jemni, Numerical simulations of particle dynamics in a poststenotic blood vessel region within the scope of extracorporeal ultrasound stenosis treatment, Medical Engineering and Physics 34 (2012) 982-989.

[11] R. Ben Haj Slama, B. Gilles, M. Ben Chiekh, J.-C. Béra, PIV for the characterization of focused field induced acoustic streaming: seeding particle choice evaluation, Ultrasonics 76 (2017) 217-226.

[12] R. Ben Haj Slama, B. Gilles, M. Ben Chiekh, J.-C. Béra, Characterization of focused-ultrasoundinduced acoustic streaming, Experimental Thermal and Fluid Science 101 (2019) 37-47.

[13] C. Poncet, S. Ferrouillat, L. Vignal, A. Memponteil, O. Bulliard-Sauret, N. Gondrexon, Enhancement of heat transfer in forced convection by using dual low-high frequency ultrasound, Ultrasonics Sonochemistry 71 (2021) 105351

[14] S. J. Lighthill, Acoustic streaming, Journal of Sound and Vibration 61 (1978) 391-418.

[15] W. L. Nyborg, Acoustic streaming due to attenuated plane waves, The Journal of Acoustical Society of America 25 (1953) 68-75.

[16] B. Moudjed, V. Botton, D. Henry, S. Millet, J.-P. Garandet, H. Ben Hadid, Oscillating acoustic streaming jet, Applied Physics Letters 105 (2014) 184102.

[17] V. Botton, J.-P. Garandet, T. Alboussière, P. Lehmann, Additional transport by oscillatory buoyancy driven convection in diffusion experiments, Journal de Physique IV 11 (2001) 57-64.

[18] E. J. Watson, Diffusion in oscillatory pipe flow, Journal of Fluid Mechanics 133 (1983) 233-244.

[19] P. C. Chatwin, On the longitudinal dispersion of passive contaminant in oscillatory flows in tubes, Journal of Fluid Mechanics 71 (1975) 513-527.

[20] M. A. Solovchuk, T. Sheu, M. Thiriet, W.-L. Lin, On a computational study for investigating acoustic streaming and heating during focused ultrasound ablation of liver tumor, Applied Thermal Engineering 56 (2013) 62-76.

[21] J. Huang, R. G. Holt, R. O. Cleveland, R. A. Roy, Experimental validation of a tractable numerical model for focused ultrasound heating in flow-through tissue phantoms, The Journal of the Acoustical Society of America 116 (2004) 2451-2458

[22] D. Thévenard, H. Ben Hadid, Low Prandtl number convection in a rectangular cavity with longitudinal thermal gradient and transverse g-jitters, International Journal of Heat and Mass Transfer 34 (1991) 2167-2173.

[23] X. Ruiz, J. Pallares, F. X. Grau, On the accuracy of the interdiffusion measurements at low and moderate Rayleigh numbers. Some computational considerations, International Journal of Heat and Mass Transfer 53 (2010) 3708-3720.

[24] M.-L. Doche, J.-Y. Hihn, A. Mandroyan, R. Viennet, F. Touyeras, Influence of ultrasound power and frequency upon corrosion kinetics of zinc in saline media, Ultrasonics Sonochemistry 10 (2003) $357-362$.

[25] E. Cooper, L. A. Coury, Jr., Mass transport in sonovoltammetry with evidence of hydrodynamic modulation from ultrasound, Journal of the Electrochemical Society 145 (1998) 1994-1999.

[26] B. Gilles, J. C. Béra, J. L. Mestas, D. Cathignol, Reduction of ultrasound inertial cavitation threshold using bifrequency excitation, Applied Physics Letters 89 (9) (2006) 094106. doi: 10.1063/1.2345230

[27] B. Moudjed, V. Botton, D. Henry, H. Ben Hadid, J.-P. Garandet, Y-shaped jets driven by an ultrasonic beam reflecting on a wall, Ultrasonics 68 (2016) 33-42.

[28] S. J. Konopka, B. McDuffie, Diffusion coefficients of ferri- and ferrocyanide ions in acqueous media, using twin-electrode thin-layer electrochemistry, Analytical Chemistry 42 (1970) 1741-1746.

[29] N. El Ghani, Modélisation expérimentale du transfert de masse en croissance cristalline par des méthodes électrochimiques en présence d'ultrasons, Ph.D. thesis, INSA Lyon (2020).

[30] F. G. Cottrell, Der reststrom bei galvanischer polarisation, betrachtet als ein diffusionsproblem, Zeitschrift für Physikalische Chemie 42U (1903) 386-431.

[31] C. Amatore, The real meaning of Nernst's steady diffusion layer concept under non-forced hy- 
drodynamic conditions. A simple model based on Levich's seminal view of convection, Journal of Electrochemical Chemistry 500 (2001) 62-70.

[32] Y. Saito, A theoretical study on the diffusion current at the stationary electrodes of circular and narrow band types, Review of Polarography 15 (1968) 177-187.

[33] Z. G. Soos, P. J. Lingane, Derivation of the chronoamperometric constant for unshielded, circular, planar electrodes, The Journal of Physical Chemistry 68 (1964) 3821-3828.

[34] B. Moudjed, V. Botton, D. Henry, H. Ben Hadid, J.-P. Garandet, Scaling and dimensional analysis of acoustic streaming jets, Physics of Fluids 26 (2014) 093602.

[35] T. Cambonie, B. Moudjed, V. Botton, D. Henry, H. Ben Hadid, From flying wheel to square flow: Dynamics of a flow driven by acoustic forcing, Physical Review Fluids 2 (2017) 123901.

670 [36] G. Launay, T. Cambonie, D. Henry, A. Pothérat, V. Botton, Transition to chaos in an acoustically driven cavity flow, Physical Review Fluids 4 (2019) 044401.

[37] T. J. Hanratty, Use of the polarographic method to measure wall shear stress, Journal of Applied Electrochemistry 21 (1991) 1038-1046.

[38] V. Sobolík, O. Wein, O. Gil, B. Tribollet, Three-segment electrodiffusion probes for measuring velocity fields close to a wall, Experiments in Fluids 9 (1990) 43-48.

[39] L. Böhm, S. Jankhah, J. Tihon, P. R. Bérubé, M. Kraume, Application of the electrodiffusion method to measure wall shear stress: Integrating theory and practice, Chemical Engineering Technology 37 (2014) 938-950. 